\title{
AVALIAÇÃO DA DIVERSIDADE GENÉTICA DE CAPSICUM SPP. COM BASE EM DESCRITORES MORFOAGRONÔMICOS E BROMATOLÓGICOS
}

\author{
EVALUATION OF THE GENETIC DIVERSITY OF CAPSICUM SPP. \\ BASED ON MORPHO AGRONOMIC AND BROMATOLOGICAL \\ DESCRIPTORS
}

\author{
${ }^{1 *}$ João Carlos Cansian Junior. \\ ${ }^{2}$ Vinicius Alves Porto Rodrigues. \\ ${ }^{3}$ Israel Felipe Gonçalves Soares. \\ ${ }^{4}$ Rafael de Almeida. \\ ${ }^{5}$ Lorena Souza Mauricio. \\ ${ }^{6}$ Felipe Cruz Paula. \\ ${ }^{7}$ Emily Suellen Fernandes Nascimento. \\ ${ }^{8}$ Rafael Nunes de Almeida. \\ ${ }^{9}$ Larissa de Carvalho Nascimento. \\ ${ }^{10}$ Fabio Gomes Zampieri. \\ ${ }^{11}$ Luciano Menini. \\ ${ }^{12}$ Monique Moreira Moulin.
}

\footnotetext{
${ }^{1}$ Instituto Federal do Espírito Santo - Campus de Alegre. E-mail: joaocj27@gmail.com ${ }^{2}$ Instituto Federal do Espírito Santo - Campus de Alegre. E-mail: viniciusbio1311@gmail.com

${ }^{3}$ Instituto Federal do Espírito Santo - Campus de Alegre. E-mail: filipeisraelgoncalves@gmail.com

${ }^{4}$ Instituto Federal do Espírito Santo - Campus de Alegre. E-mail: rafael.t.dealmeida@gmail.com

${ }^{5}$ Instituto Federal do Espírito Santo - Campus de Alegre. E-mail: lorena_rittberg@hotmail.com ${ }^{6}$ Instituto Federal do Espírito Santo - Campus de Alegre. E-mail: felipe.cpaula64@gmail.com

${ }^{7}$ Instituto Federal do Espírito Santo - Campus de Alegre. E-mail: emilysuellencbl@gmail.com

${ }^{8}$ Universidade Estadual do Norte Fluminense Darcy Ribeiro. E-mail: almeida.rna94@gmail.com

${ }^{9}$ Instituto Federal do Espírito Santo - Campus de Alegre. E-mail: larissacn1@hotmail.com

${ }^{10}$ Instituto Federal do Espírito Santo - Campus de Alegre. E-mail: fgzampieri@hotmail.com

${ }^{11}$ Instituto Federal do Espírito Santo - Campus de Alegre. E-mail: lmenini@ifes.edu.br

${ }^{12}$ Instituto Federal do Espírito Santo - Campus de Alegre. E-mail: moniquemoulin@gmail.com

*Autor de correspondência
}

Artigo submetido em 02/04/2021, aceito em 11/06/2021 e publicado em 30/07/2021.

Resumo: O gênero Capsicum pertence à família Solanaceae e possui grande diversidade evidenciada entre 38 espécies já catalogadas, todavia apenas cinco espécies são domesticadas, $C$. annuum, $C$. baccatum, $C$. pubescens, $C$. frutescens e $C$. Chinense. As pimentas possuem uma grande variabilidade fenotípica, principalmente em relação à coloração, forma e tamanho dos frutos, que pode ser analisada através de descritores morfoagronômicos, auxiliando nos estudos de diversidade genética da cultura. Objetivou-se com este estudo caracterizar com base em descritores morfoagronômicos e bromatológicos, 33 acessos de pimentas do banco de germoplasma do IFES Campus de Alegre e, com isso, estimar a divergência genética deles. Foram selecionados 22 descritores morfoagronômicas e 5 avaliações físico-químicas. Estimou-se a contribuição relativa das características através do método de 
Singh e a dissimilaridade genética entre os acessos empregando a distância de Mahalanobis e o método Unweighted Paired Group Methodusing Arithmetic Averages (UPGMA). O dendrograma de dissimilaridade foi composto por nove grupos e revelou que os acessos IFES58 e IFES78 são os que apresentam maior similaridade, enquanto os genótipos IFES58 e IFES96 foram os mais distantes geneticamente. As caracterizações morfoagronômica e físico-química foram eficientes para estimar a diversidade genética entre os 33 acessos de pimenta e destacar acessos com potencial para exploração em diferentes nichos de mercado para consumo in natura, processado ou para fins ornamentais. $\mathrm{O}$ estudo realizado evidencia a existência de alta variabilidade genética entre os acessos de Capsicum do banco de germoplasma do IFES Campus de Alegre.

Palavras-chave: banco ativo de germoplasma; caracterização físico-química; caracterização morfológica; pré-melhoramento.

\begin{abstract}
The genus Capsicum belongs to the Solanaceae family and has great diversity, evidenced among 38 species already cataloged, however only five species are domesticated, C. annuum, $C$. baccatum, C. pubescens, C. frutescens and C. Chinense. Peppers have a great phenotypic variability, mainly in relation to color, shape and size of the fruits, which may be analyzed through morphoagronomic descriptors, helping the studies about genetic diversity of the crop. The aim of this study was to characterize 33 accessions of peppers from the germplasm bank of the IFES Campus de Alegre, and also estimate the genetic divergence between them, based on morphoagronomic and bromatological descriptors, .Twenty-two morphoagronomic descriptors and five physicochemical evaluations were selected. The relative contribution of the traits was estimated using the Singh method and the genetic dissimilarity between accessions using the Mahalanobis distance and the Unweighted Paired Group Methodusing Arithmetic Averages (UPGMA) method. The dissimilarity dendrogram was composed of nine groups and revealed that the IFES58 and IFES78 accessions are the ones with the greatest similarity, while the IFES58 and IFES96 genotypes were the most distant genetically. The morphoagronomic and physicochemical characterizations were efficient to estimate the genetic diversity among the 33 pepper accessions and highlight accessions with potential for exploration in different market niches, for fresh consumption, processed or for ornamental purposes. The study carried out evidences of the existence of high genetic variability among accessions of Capsicum from the germplasm bank of the IFES Campus de Alegre.
\end{abstract}

Keywords: active germplasm bank; physicochemical characterization; morphological characterization; pre-improvement.

\section{INTRODUÇÃO}

O gênero Capsicum inclui as pimentas e pimentões (família Solanaceae), possui grande variabilidade genética, é composto por 38 espécies catalogadas, sendo apenas cinco espécies domesticadas, são elas: C. annuum, C. baccatum, $C$. pubescens, $C$. frutescens e $C$. chinense (BIANCHI et al., 2016; JACQZAMLJEN; ZUPANC; SLATNAR, 2020; MOREIRA et al., 2018).

O Brasil é considerado um dos principais centros de diversidade tanto de espécies silvestres quanto de espécies domesticadas, dentre estas últimas a $C$. chinense é tida como a mais significativa no país, pois além de haver indícios da Bacia Amazônica ser seu centro de origem, a mesma pimenta é também a mais cultivada e consumida no país (BABA et al., 2016; MOREIRA et al., 2018; ULHOA et al., 2017).

Algumas espécies de pimenta, como C. annuum, foram domesticadas entre seis e nove mil anos atrás na região que hoje corresponde ao México e fazia parte da dieta dos ameríndios (SILVA et al., 2015). Estima-se que cerca de $25 \%$ da população mundial consuma pimenta diariamente, 
principalmente na forma in natura, desidratada ou em molho (ANTONIO; WIEDEMANN; VEIGA JUNIOR, 2018; KANTAR et al., 2016).

A pimenta é uma das culturas mais difundidas ao redor do mundo, com uma produção superando os 30 milhões de toneladas anualmente (PEREIRA-DIAS et al., 2017) O gênero possui inúmeras aplicações comerciais, principalmente na indústria de cosméticos, farmacêutica, culinária e decoração (FERRAZ et al., 2016). A pimenta tem relevância socioeconômica significativa e é cultivada em todo o Brasil principalmente por agricultores familiares (CARVALHO et al., 2015).

A pungência é a característica mais marcante das pimentas e está relacionada a presença de capsaicinoides no fruto, especialmente na placenta, e por conta disso as mesmas são largamente consumidas ao redor do mundo, além de apresentarem outros compostos benéficos à saúde como carotenóides, polifenóis, peptídeos, flavonoides, vitaminas B1, B2, A, C, E, minerais como cálcio, potássio, ferro, fósforo e diversos outros compostos que possuem ação antioxidante, antimicrobiana, anti-inflamatória e até mesmo anticancerígena (BAENAS et al., 2019; JEEATID et al., 2018; PAULUS et al., 2015; SARPRAS et al., 2019; SHAMYA AROKIA RAJAN et al., 2020).

A capsaicina e dihidrocapsaicina são os principais capsaicinoides responsáveis por grande parte do sabor picante das pimentas (JEEATID et al., 2018). A capsaicina é sintetizada exclusivamente pelos membros do gênero Capsicum e desempenha ação analgésica, antiobesidade, anti-hipertensiva e gastroprotetora (HAN et al., 2019). Esse fitoquímico também pode incentivar a produção de saliva e enzimas digestivas, desenvolver atividade anticoagulante entre outras propriedades (SANATI; RAZAVI; HOSSEINZADEH, 2018). Os capsaicinoides são produzidos como um metabólito secundário da pimenta, em que provavelmente a mesma desenvolveu os capsaicinoides com a finalidade de evitar a ação de fungos, bactérias e herbívoros, fenômeno conhecido como coevolução (SRINIVASAN, 2016).

A concentração de compostos bioativos e de nutrientes variam em razão da cultivar da pimenta, do estágio de amadurecimento, das condições ambientais, da parte do fruto e outros fatores (BAENAS et al., 2019). A pimenta também é uma planta medicinal empregada no tratamento de tosse, dor de dente e de garganta, artrite, reumatismo, cicatrização de feridas, estimulador de apetite, diabetes, doenças cardiovasculares e várias outras funções (BOGUSZ et al., 2018; LOIZZO et al., 2015; PAULUS et al., 2015; SANATI; RAZAVI; HOSSEINZADEH, 2018).

As pimentas possuem uma grande variabilidade fenotípica, principalmente em relação à coloração, forma e tamanho dos frutos; essa variabilidade pode ser analisada através de descritores morfoagronômicos, que auxiliam nos estudos de diversidade genética da cultura (BIANCHI et al., 2016). Em razão disso, objetivou-se com o presente trabalho caracterizar 33 acessos de pimentas do banco de germoplasma do IFES Campus de Alegre por intermédio de descritores morfoagronômicos e bromatológicos e, com isso, estimar a divergência genética existente.

\section{MATERIAIS E MÉTODOS}

O experimento foi conduzido no Setor de Agroecologia do Instituto Federal de Educação Ciência e Tecnologia do Espírito Santo (IFES), no município de Alegre-ES, latitude $20^{\circ} \quad 45^{\prime} \quad 20^{\prime \prime} \mathrm{S}$ e longitude $41^{\circ} 27^{\prime} 43^{\prime \prime} \mathrm{O}$ a $131 \mathrm{~m}$ de altitude. Foram avaliados 33 genótipos de pimenta do Banco de Germoplasma do IFES Campus de Alegre.

A semeadura foi realizada em tubetes e o substrato empregado continha uma 
mistura de $60 \%$ de terra e $40 \%$ de material orgânico. Após 50 dias foi realizado o transplantio para condições de campo, utilizando-se delineamento em Blocos Casualizados (DBC), com quatro repetições e espaçamento de $0,8 \mathrm{~m}$ entre linhas e $0,4 \mathrm{~m}$ entre plantas. A unidade experimental foi composta por uma única planta. Foram avaliadas 12 variáveis quantitativas e 10 qualitativas, da lista de descritores morfológicos sugerida pelo Bioversity International (IPGRI, 1995).

Os descritores qualitativos analisados foram: hábito de crescimento da planta (HC), forma da folha (FF), forma do caule (FC), cor do fruto no estágio intermediário (CFREI), cor do fruto no estágio maduro (CFREM), forma do fruto (FFR), forma da ponta do fruto (FPFR), superfície do fruto (SFR), forma da base do fruto (FBFR) e ondulação transversal do fruto (OTF).

$\mathrm{E}$ os descritores quantitativos avaliados foram: altura da planta (AP), diâmetro da copa (DCO), comprimento do pedúnculo (CP), diâmetro do caule (DCA), comprimento do fruto (CFR), diâmetro do fruto (DFR), comprimento da folha (CF), largura da folha (LF) espessura do pericarpo (EP), peso do fruto (PFR), número de lóculos (NL) e número de semente por fruto (NSFR).

Foram também avaliadas cinco variáveis físico-químicas quantitativas de fruto: sólidos solúveis totais (SST), acidez total titulável (ATT), umidade (UM), teor de cinzas (TC) e pH. As análises foram realizadas no Laboratório de Química Aplicada do IFES Campus de Alegre. Os sólidos solúveis totais ( ${ }^{\circ} \mathrm{Brix}$ ) foram obtidos com a adição de duas gotas do suco de pimenta, no refratômetro manual (VILAS BOAS, 2008).

$\mathrm{O} \mathrm{pH}$ dos frutos foi quantificado com o auxílio de um pHmetro manual, com a adição de $1 \mathrm{~mL}$ de suco de pimenta e 20 $\mathrm{mL}$ de água destilada em um béquer, e posteriormente foi aferido o $\mathrm{pH}$ (VILAS BOAS, 2008). A porcentagem de umidade das amostras, o teor de cinzas da amostra integral e a acidez total titulável que utilizou o titulante $\mathrm{NaOH}$ a $0,01 \mathrm{M}$, para a determinação da porcentagem de ácido cítrico, foram realizadas de acordo com o recomendado pelo Instituto Adolfo Lutz (BRASIL, 2008).

A colinearidade entre acessos foi realizada com o auxílio do software $R$ utilizando o pacote (ggplot2), onde devido a algumas características apresentarem colinearidade (diâmetro da copa, diâmetro do fruto e $\mathrm{pH}$ ), das 27 características avaliadas, apenas 24 foram utilizadas para a formulação das análises. As demais análises foram realizadas com auxílio do programa Genes (CRUZ, 2006).

O método de Singh (1981) foi utilizado para determinar a contribuição relativa das características. As estimativas da matriz de distância genética das variáveis quantitativas foram obtidas com base na distância de Mahalanobis.

Figura 1: Variabilidade fenotípica dos frutos de pimenta do Banco de Germoplasma do IFES Campus de Alegre.

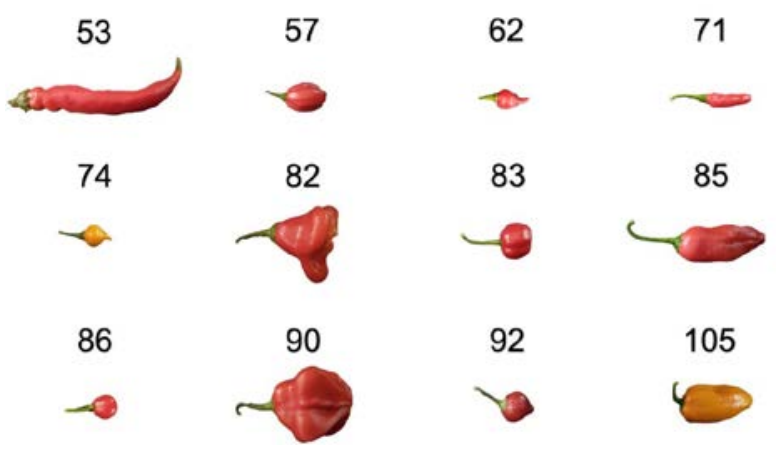

Fonte: os autores (2021).

O agrupamento dos acessos foi obtido pelo método Unweighted Paired Group Methodusing Arithmetic Averages (UPGMA) e a validação pelo coeficiente de correlação cofenético. E em relação às variáveis qualitativas, utilizou-se uma metodologia de análise descritiva em percentual da frequência das classes fenotípicas. 


\section{RESULTADOS E DISCUSSÃO}

O método de Singh (1981), que foi usado para mensurar a contribuição relativa das características para a variabilidade genética, demonstrou que a variável CFR contribuiu com $23 \%$ da diversidade genética entre os acessos, apresentando valores de 13,03 a 1,22 cm. As características PFR e SST contribuíram com $19 \%$ e $16 \%$ para o estudo de diversidade e apresentaram valores de 0,80 a 24,37g e 3,60 a 13,00 ${ }^{\circ}$ Brix, respectivamente (Figura 2 ).

Figura 2. Contribuição relativa das características quantitativas para diversidade genética entre os 33 genótipos de Capsicum spp. pelo método de SINGH (1981).

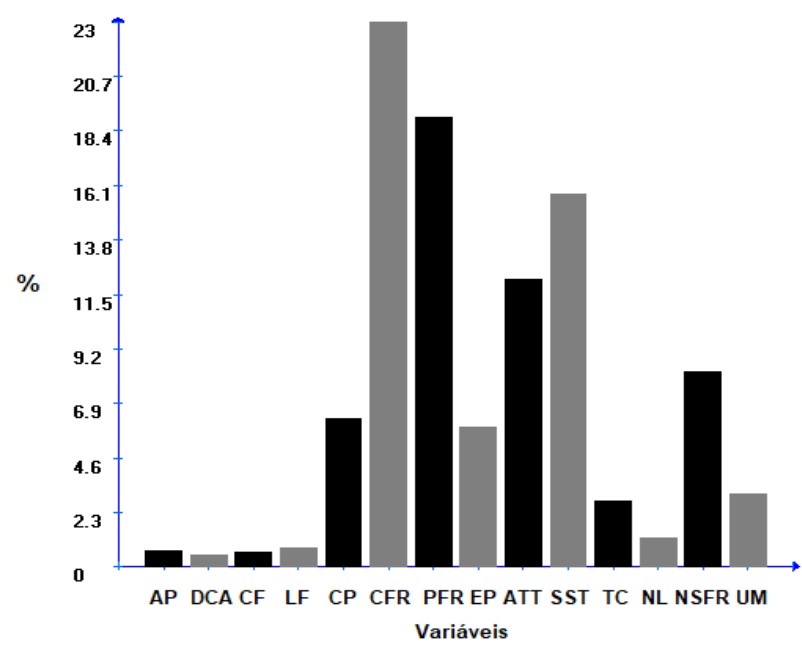

Fonte: os autores (2021).

COSTA et al. (2020), ao quantificarem a diversidade genética de 14 genótipos de Capsicum spp. do município de Floriano-PI, constataram que uma das características que mais contribuíram para a discriminação dos acessos foi o peso do fruto. THUL et al. (2009), ao estudarem a divergência fenotípica de seis espécies de Capsicum baseando-se em características relacionadas à produção, observaram que o comprimento do fruto foi a característica que mais contribuiu para a divergência genética. Os resultados obtidos por esses autores são similares aos obtidos nesse estudo, o que demonstra o potencial discriminatório das características para estudos de diversidade genética.

Com a utilização do método hierárquico de agrupamento UPGMA houve formação de nove grupos, sendo realizado um corte a uma distância de 0,24 no dendrograma em razão de sua topologia. A correlação cofenética demonstrou um valor de 0,72, sendo considerada satisfatória segundo SANTOS et al. (2019). A correlação cofenética é essencial para a elaboração de interpretações mais sólidas a respeito da divergência entre os acessos (ROHLF, 1970).

A distância de Mahalanobis, adotada como medida de dissimilaridade revelou que os acessos IFES58 e IFES78 são os que apresentam maior similaridade, enquanto os genótipos IFES58 e IFES96 foram os que apresentaram um maior distanciamento para as características avaliadas (Figura 3).

Genótipos reunidos em grupos de maior dissimilaridade podem ser considerados como promissores em programas de cruzamentos artificiais, sendo também necessário que os genitores apresentem alta associação e variabilidade nas características que serão melhoradas, uma vez que o melhoramento genético atua sobre a variabilidade disponível em um banco de germoplasma (DE MELO et al., 2019).

Figura 3: Dendrograma de dissimilaridade genética, obtido com base em descritores quantitativos e qualitativos de Capsicum, entre 33 acessos de Pimenta do Banco de Germoplasma do IFES Campus de Alegre. 


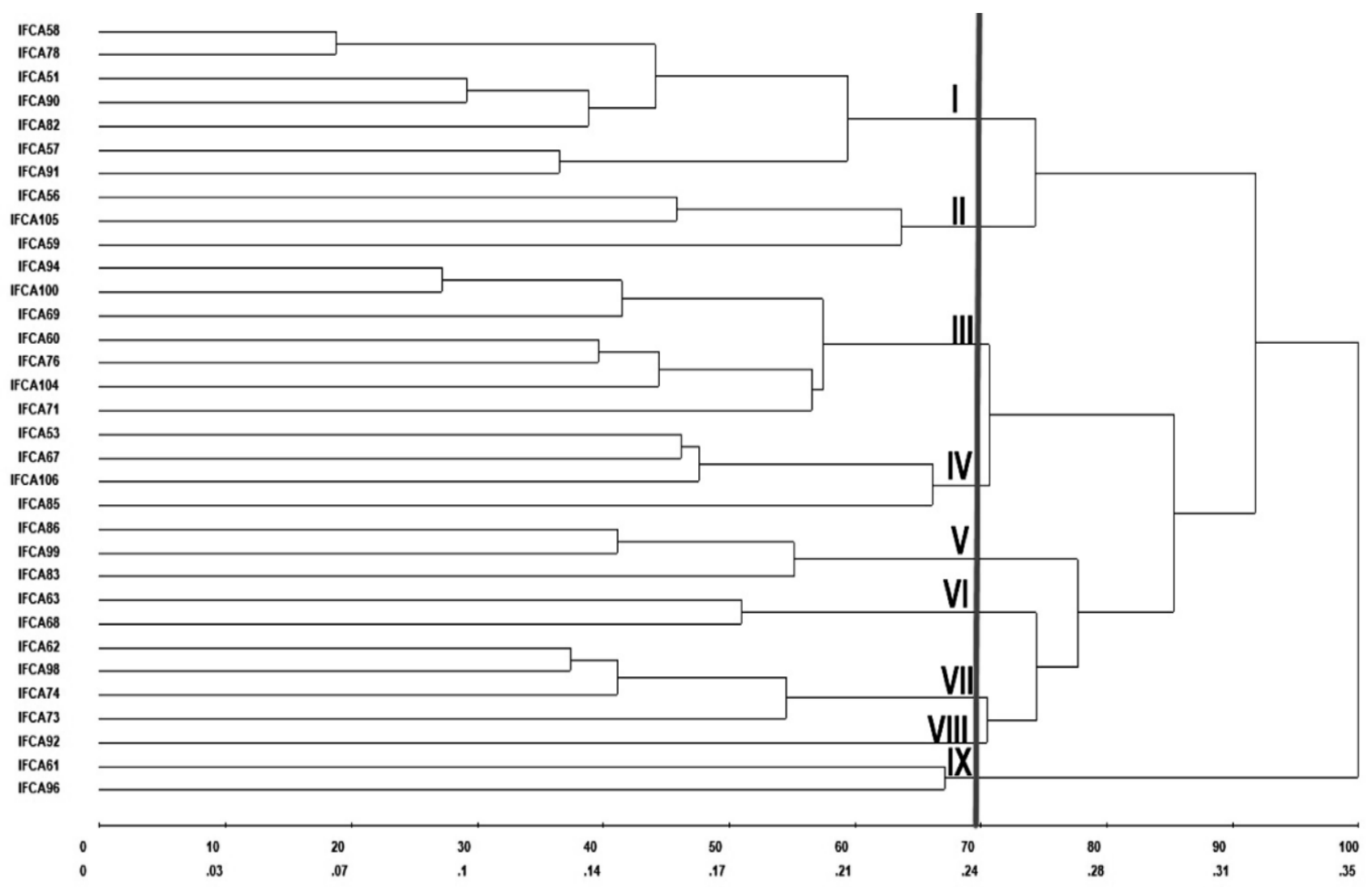

Fonte: os autores (2021).

O grupo I, constituído pelos acessos IFES51, IFES57, IFES58, IFES78, IFES82, IFES90 e IFES91, foi caracterizado por acessos com os maiores teores de umidade (variando de 86,86 a 92,15\%), valores com baixa acidez total titulável (0,25 a 0,33\%), predominância de superfície do fruto semi rugosa e hábito de crescimento intermediário.

Essas características fazem desses acessos mais indicados para a comercialização, principalmente pela característica acidez total titulável, pois menores valores para esta característica indica uma maior durabilidade do fruto, por conta de o mesmo apresentar um melhor estado de conservação, o que proporciona qualidade superior ao produto final (BORGES et al., 2015; SANTOS et al., 2020). A acidez total titulável é considerada um critério relevante na determinação do estado de conservação de um alimento, pois retarda o processo de decomposição, seja ele por fermentação, hidrólise ou oxidação (BORGES et al., 2015).

No grupo II, ficaram alocados os acessos IFES56, IFES59 e IFES105, que foram caracterizados por maiores comprimentos (variando de 84,74 a 89,31 $\mathrm{mm})$ e larguras foliares (44,54 a 51,55 $\mathrm{mm}$ ), além de altos teores de umidade $(88,88$ a $90,39 \%)$, forma da base do fruto raso e forma da ponta do fruto redondo. Devido a maior área foliar, esses acessos podem apresentar uma maior taxa fotossintética, que é essencial para o desenvolvimento da planta (COSTA et al., 2020). Em certas condições, a maior área foliar pode estimular de forma considerável a produção de biomassa, consequentemente aumentando a taxa de crescimento da planta (ÁVILA; BARBOSA, 2019; PAULUS et al., 2015).

O grupo III compreendeu um dos maiores números de acessos, correspondendo a sete acessos ou 21,21\% do total. Os genótipos IFES60, IFES69, IFES71, IFES76, IFES94, IFES100 e 
IFES104 foram agrupados por possuírem, médios valores de umidade (variando de 79,49 a 90,17\%), frutos pontiagudos, alongados e verdes no estágio intermediário. A variável umidade está associada com a composição e qualidade do fruto, influenciando diretamente no processamento e armazenamento do mesmo (REBOUÇAS; VALVERDE; TEIXEIRA, 2013).

O grupo IV, constituído pelos genótipos IFES53, IFES67, IFES85 e IFES106, foi discriminado por acessos com maiores comprimentos de fruto (6,90 a $11,67 \mathrm{~cm}$ ), elevados valores para número de sementes por fruto (variando de 60,50 a 97,50) e valores medianos para o diâmetro do caule (1,41 a 1,65 cm). Estes genótipos que apresentam frutos grandes podem ser consumidos in natura ou desidratados, enquanto frutos pequenos são preferencialmente consumidos na forma de molhos industrializados (SILVA et al., 2021).

O maior número de sementes por fruto é uma característica relevante, tanto para a reprodução e multiplicação das pimentas quanto para a comercialização das sementes, em que pode ser uma fonte de renda alternativa aos produtores, considerando que algumas variedades do gênero Capsicum produzem poucas sementes (CRUZ et al., 2019; COSTA et al., 2020; PAULUS et al., 2015).

O diâmetro do caule é um aspecto que deve ser considerado ao cultivar uma variedade de pimenta, em especial as ornamentais, pois plantas com menores diâmetros de caule tendem a tombar e a perder muitos frutos, gerando prejuízo aos produtores (BIANCHI et al., 2016). Ainda sobre o grupo IV, notou-se uma predominância da superfície rugosa dos frutos, em função disso esses frutos têm uma maior rejeição na comercialização in natura, em razão dos consumidores compará-los com produtos em início de decomposição (DOMENICO et al., 2012).

$\mathrm{O}$ grupo $\mathrm{V}$ reuniu os genótipos

IFES83, IFES86 e IFES99, que apresentaram maiores valores para número de lóbulos $(3,25$ a 3,50) e sólidos solúveis totais $\left(9,40\right.$ a $\left.11,75{ }^{\circ} \mathrm{Brix}\right)$, cor vermelha do fruto maduro, forma do fruto quase redondo, além dos mais baixos valores para o comprimento do fruto (1,30 a 2,15 $\mathrm{cm})$. Esta última característica é atraente para os produtores, pois apesar de a colheita à mão ser mais trabalhosa, detêm de maior preço no comércio (COSTA et al., 2020).

Por apresentarem um teor de sólidos solúveis elevado, os acessos componentes do grupo $\mathrm{V}$ são mais indicados para a indústria, pois maiores concentrações de sólidos solúveis totais resultam em um maior rendimento e beneficiamento do fruto, principalmente na indústria de alimentos (BORGES et al., 2015).

No grupo VI ficaram alocados os acessos IFES63 e IFES68, os quais foram agrupados por possuírem menor número de sementes por fruto $(19,00$ a 35,25$)$, reduzido peso do fruto (2,03 a 2,30g), superfície do fruto lisa e menor altura (31,68 a $40 \mathrm{~cm})$. A variável altura da planta é essencial para indicar se determinados genótipos demandam de muita ou pouca mão de obra, ou de algum tipo de tutoramento (SILVA et al., 2021). Além disso, plantas com menor porte são mais indicadas no melhoramento de variedades ornamentais (SILVA et al., 2021).

O grupo VII, representado pelos genótipos IFES62, IFES73, IFES74 e IFES98, foi discriminado pelos acessos com médios resultados para as características de largura da folha (variando de 30,44 a 40,63 mm), baixo comprimento do pedúnculo (1,69 a 2,05 $\mathrm{cm})$, superfície do fruto lisa, coloração do fruto maduro variando do amarelo ao vermelho e porte da planta relativamente baixo (40 a $55 \mathrm{~cm}$ ).

Essas características fazem desses acessos mais indicados para a ornamentação, devido principalmente à característica cor dos frutos, pois variedades de pimentas que possuem flores 
e frutos com coloração diversificada e porte baixo são mais apreciadas para ornamentação (SUDRÉ et al., 2005). Além disso, destaca-se o baixo valor para o caráter comprimento do pedúnculo, que pode ser um empecilho para os produtores, já que pedúnculos com menores comprimentos se destacam mais dificilmente (COSTA et al., 2020).

O grupo VIII foi formado apenas pelo acesso IFES92, que se caracterizou por apresentar valores médios para as características acidez (0,43\%), sólidos solúveis totais $\left(8,90^{\circ} \mathrm{Brix}\right)$ e peso do fruto $(6,52 \mathrm{~g})$ e forma do fruto quase redondo.

O grupo IX reuniu os genótipos IFES61 e IFES96, que apresentavam formato triangular de fruto, baixos valores para comprimento do fruto (2,08 a 2,38 $\mathrm{cm}$ ) e espessura do pericarpo (variando de 0,44 a $0,57 \mathrm{~mm}$ ). Plantas com tamanhos de frutos pequenos apresentam melhor aceitação no mercado ornamental de pimentas (DE MELO et al., 2014). Porém, este grupo há uma desvantagem em relação a menor espessura do pericarpo, visto que frutos com pericarpos delgados podem diminuir a resistência da cultivar contra organismos patogênicos no armazenamento (BIANCHI et al., 2020).

O formato do caule predominante foi angular, característica também observada no estudo de SILVA et al. (2021), em que realizaram a caracterização morfológica de 21 acessos da coleção de pimentas do CCAA/UFMA, no estado do Maranhão. Estes mesmos autores encontraram resultados semelhantes com a do presente estudo em relação à forma da folha, apresentando as formas deltoide, lanceolada e oval, sendo esta última observada com maior frequência.

As cores dos frutos no estádio intermediário e maduro foram predominantemente verde e vermelha, respectivamente. BIANCHI et al. (2020), ao realizarem caracterização morfoagronômica de 55 acessos de $C$. chinense oriundas de quatro regiões do Brasil e um acesso do Peru, relataram a cor laranja como a mais comum em frutos no estágio intermediário e vermelha como a mais comum em frutos maduros.

O hábito de crescimento variou entre o ereto e o intermediário. Resultados contrastantes foram encontrados por RAHMAN, HOSSAIN, AFROZ (2017), em que $80 \%$ das plantas tiveram hábito prostrado de um total de 60 acessos de pimentas caracterizados por descritores morfológicos, em um banco ativo de germoplasma (BAG) de Bangladesh.

BIANCHI et al. (2016) caracterizaram 30 acessos de pimentas do gênero Capsicum do banco ativo de germoplasma do IFES Campus de Alegre, em relação à forma do fruto obteve a forma alongada como a mais predominante entre os acessos, similar ao obtido no presente estudo.

\section{CONCLUSÕES}

As caracterizações morfoagronômica e físico-química foram eficientes para estimar a diversidade genética entre os 33 acessos de pimenta e destacar acessos com potencial para atender diferentes nichos de mercado e também para serem empregados em futuros programas de melhoramento.

Os acessos poderão ser distribuídos em nove diferentes grupos de dissimilaridade, havendo grupos com potencial para exploração de pimentas para consumo in natura ou desidratado (grupo IV), processado (grupo V) e para fins ornamentais (grupos VII).

$\mathrm{O}$ estudo realizado evidencia a existência de alta variabilidade genética entre os acessos de Capsicum do banco de germoplasma do IFES Campus de Alegre.

\section{AGRADECIMENTOS}

Agradeço ao IFES Campus de Alegre e ao CNPq pela bolsa de iniciação científica concedida. 


\section{REFERÊNCIAS}

ALVARENGA, Marco Antonio Alvarenga Rezende (Ed.). Tomate: produção em campo, em casa-de-vegetação e em hidroponia. UFLA, 2004.

ANTONIO, A. S.; WIEDEMANN, L. S. M.; VEIGA JUNIOR, V. F. The genus: Capsicum: a phytochemical review of bioactive secondary metabolites. RSC Advances, v. 8, n. 45, p. 25767-25784, 2018.

ÁVILA, M. DE S. N.; BARBOSA, J. M.Bean pepper growth analysis at different levels of solar radiation. Brazilian Journal of Development, v. 5, n. 12, p. 31985-31997, 2019.

BABA, V. Y. et al. Genetic diversity of Capsicum chinense accessions based on fruit morphological characterization and AFLP markers. Genetic Resources and Crop Evolution, v. 63, n. 8, p. 1371-1381, 2016.

BAENAS, N. et al. Industrial use of pepper (Capsicum annum L.) derived products: Technological benefits and biological advantages. Food Chemistry, v. 274, p. 872-885, 2019.

BIANCHI, P. A. et al. Morphological characterization and analysis of genetic variability among pepper accessions. Ciencia Rural, v. 46, n. 7, p. 1151-1157, 2016.

BIANCHI, P. A. et al. Biomorphological characterization of Brazilian Capsicum chinense Jacq. Germplasm. Agronomy, v. 10, n. 3, p. 1-17, 2020.

BOGUSZ, S. et al. Brazilian Capsicum peppers: capsaicinoid content and antioxidant activity. Journal of the Science of Food and Agriculture, v. 98, n. 1, p. 217-224, 2018.
BORGES, K. M. et al. Morpho-agronomic and physicochemical characterisation of the pepper for the State of Roraima.

RevistaAgro@Mbiente on-Line, v. 9, n. 3, p. 292, 2015.

BRASIL - Instituto Adolfo Lutz. (2008). Métodos físico-químicos para análise de alimentos. $4^{\mathrm{a}}$ ed. $1^{\mathrm{a}}$ ed. Digital, São Paulo, p.1020.

BRILHANTE, B. D. G.

\section{CARACTERIZAÇÃO MORFOAGRONÔMICA, BROMATOLÓGICA E MOLECULAR DE GERMOPLASMA DE Capsicum spp. Alegre: UFES, 2018.}

CRUZ, D. R. C. et al. Avaliação morfológica de genótipos de pimentas bode desenvolvidos no IF Goiano Campus Ceres. Revista Brasileira Multidisciplinar, v. 22, n. 3, p. 2, 2019.

CARVALHO, S. I. C. et al. Transferability of microsatellite markers of Capsicum annuum L. To C. frutescens L. and C. Chinense Jacq. Genetics and Molecular Research, v. 14, n. 3, p. 7937-7946, 2015.

COSTA, L. DA S. et al. Characterization of Capsicum Spp. By Multivariate Techniques in Southern Piauí. Brazilian Journal of Development, v. 6, n. 12, p. 97371-97385, 2020.

CRUZ, C. D. Programa GENES: estatística experimental e matrizes. Viçosa: UFV. 285p. 2006.

DAMBROS, J. I. Estabilidade de compostos potencialmente bioativos $e$ alterações de qualidade em frutos e produtos de pimenta (Capsicum spp.). UNIVERSIDADE FEDERAL DE PELOTAS, 2014.

DE MELO, L. F. et al. Potencial ornamental de acessos de pimenta. Ciencia Rural, v. 44, n. 11, p. 2010-2015, 2014. 
DOMENICO, C. I. et al. Caracterização agronômica e pungência em pimenta de cheiro. Horticultura Brasileira, v. 30, n. 3, p. 466-472, 2012.

FERRAZ, R. M. et al. Caracterização morfoagronômica preliminar de acessos de pimentas cumari. Horticultura Brasileira, v. 34, n. 4, p. 498-506, 2016.

HAN, K. et al. A MYB transcription factor is a candidate to control pungency in Capsicum annuum. Theoretical and Applied Genetics, v. 132, n. 4, p. 12351246, 2019.

IPGRI (International Plant Genetic Resources Institute). Descriptors for Capsicum (Capsicum spp.). International Plant Genetic Resources Institute, Rome, Italy, p.49, 1995.

JACQZAMLJEN, T.; ZUPANC, V.; SLATNAR, A. Influence of irrigation on yield and primary and secondary metabolites in two chilies species, Capsicum annuum L. and Capsicum chinense. Agricultural Water Management, v. 234, n. 2, p. 106104, 2020.

JEEATID, N. et al. Influence of water stresses on capsaicinoid production in hot pepper (Capsicum chinense Jacq.) cultivars with different pungency levels. Food Chemistry, p. 792-797, 2018.

KANTAR, M. B. et al. Vitamin variation in capsicum spp. Provides opportunities to improve nutritional value of human diets.

PLoS ONE, v. 11, n. 8, p. 1-12, 2016.

LOIZZO, M. R. et al. Evaluation of chemical profile and antioxidant activity of twenty cultivars from Capsicum annuum, Capsicum baccatum, Capsicum chacoense and Capsicum chinense: A comparison between fresh and processed peppers.
LWT - Food Science and Technology, v. 64, n. 2, p. 623-631, 2015.

MELO, A. V. DE et al. Divergência genética entre híbridos de milho em condições de deficiência hídrica. Revista de Agricultura Neotropical, v. 6, n. 3, p. 66-75, 2019.

MOREIRA, A. F. P. et al. Genetic diversity, population structure and genetic parameters of fruit traits in Capsicum chinense. Scientia Horticulturae, v. 236, p. 1-9, 2018.

MOULIN, M. M. et al. Genetic dissection of agronomic traits in capsicum baccatum var. Pendulum. Genetics and Molecular Research, v. 14, n. 1, p. 2122-2132, 2015.

OROBIYI, A. et al. Agro-morphological characterization of chili pepper landraces (Capsicum annuum L.) cultivated in Northern Benin. Genetic Resources and Crop Evolution, v. 65, n. 2, p. 555-569, 2018.

PAULUS, D. et al. Crescimento, produção e qualidade de frutos de pimenta (Capsicum annuum) em diferentes espaçamentos. Horticultura Brasileira, v. 33, n. 1, p. 91-100, 2015.

PEREIRA-DIAS, L. et al. Use of molecular markers to assist the development of inbred lines under open field conditions: The case of criollo peppers (Capsicum annuum L.) from Mexico. Notulae Botanicae Horti Agrobotanici Cluj-Napoca, v. 45, n. 2, p. 365-368, 2017.

RAHMAN, S.; HOSSAIN, M.; AFROZ, R. Morphological characterization of chilli germplasm in Bangladesh. Bangladesh Journal of Agricultural Research, v. 42, n. 2, p. 207-219, 2017.

REBOUÇAS, T. N. H.; VALVERDE, R. M. V.; TEIXEIRA, H. L. Bromatologia da 
pimenta malagueta in natura e processada em conserva. Horticultura Brasileira, v. 31, n. 1, p. 163-165, 2013.

SANATI, S.; RAZAVI, B. M.; HOSSEINZADEH, $\mathrm{H}$. A review of the effects of Capsicum annuum L. And its constituent, capsaicin, in metabolic syndrome. Iranian Journal of Basic Medical Sciences, v. 21, n. 5, p. 439-448, 2018.

SANTOS, B. C. et al.Stability of malagueta peppers (Capsicum Frutescens) in canned during ambient temperature storage. Brazilian Journal of Development, v. 6, n. 8, p. 56214-56231, 2020.

SANTOS, T. DE O. et al. Characterization and Diversity of Peppers (Capsicum spp.) Genotypes Based on Morphological Traits Using Multivariate Analysis. Journal of Experimental Agriculture International, v. 39, n. 1, p. 1-10, 2019.

SARPRAS, M. et al. Comparative analysis of developmental changes of fruit metabolites, antioxidant activities and mineral elements content in Bhut jolokia and other Capsicum species. LWT - Food Science and Technology, v. 105, p. 363370, 2019.

SCOTT, A. J; KNOTT, M. A. Cluster analysismethods for groupingmeans in the analysis of variance. Biometrics, $\mathrm{v}$. 30, p. 507-512, 1974.

SHAMYA AROKIA RAJAN, M. et al. Effect of seaweed on seed germination and biochemical constituents of Capsicum annuum. Biocatalysis and Agricultural Biotechnology, v. 29, p. 101761, 2020.

SILVA, H. W. DA et al. Higroscopicidade das sementes de pimenta (Capsicum chinense L.). Revista Brasileira de Engenharia Agrícola e Ambiental, v. 19, n. 8, p. 780-784, 2015.
SILVA, J. M. et al. Morphological characterization of accessions of peppers ( Capsicum spp .) Preserved in the state of Maranhão. Brazilian Journal of Development, v. 7, n. 3, p. 21358-21373, 2021.

SOLOMON, A. M. et al. Genetic diversity and population structure of Ethiopian Capsicum germplasms. PLoS ONE, v. 30, n. 3, p. 1-20, 2019.

SRINIVASAN, K. Biological Activities of Red Pepper (Capsicum annuum) and Its Pungent Principle Capsaicin: A Review. Critical Reviews in Food Science and Nutrition, v. 56, n. 9, p. 1488-1500, 2016.

SUDRÉ, C. P. et al. Divergência genética entre acessos de pimenta e pimentão utilizando técnicas multivariadas.

Horticultura Brasileira, v. 23, n. 1, p. 22-27, 2005.

THUL, S. T. et al. Estimation of phenotypic divergence in a collection of Capsicum species for yield-related traits. Euphytica, v. 168, n. 2, p. 189-196, 2009.

ULHOA, A. B. et al. Obtenção e caracterização morfoagronômica de linhagens de pimenta do tipo Jalapeño Amarelo. Horticultura Brasileira, v. 35, n. 3, p. 343-348, 2017.

VASCONCELOS, C. S. et al. Distância genética entre variedades crioulas de Capsicum chinense. Genetic distance in Capsicum chinense landraces. Magistra, v.26, n.2, p.178-185, 2014.

VILAS BOAS, E.V.B. et al. Medidas de Qualidade. Universidade Federal de Lavras. Lavras. 2008. 